\title{
Adaptación Transcultural y Evaluación de las Estructuras Factoriales del Test de Adicción a Internet en Chile: Desarrollo de una Versión Abreviada
}

\author{
A Chilean Transcultural Adaptation and Assessment of the Internet Addiction Test's \\ Factor Structures: Development of a Brief Version
}

\author{
Cristóbal Hernández Contreras ${ }^{1}$ y Diana Rivera Ottenberger ${ }^{2}$
}

\begin{abstract}
Resumen
La Adicción a Internet es un fenómeno clínico que ha dado señales de alarma sobre las propiedades adictivas de las nuevas tecnologías de la información y comunicación (TICs). El objetivo del estudio fue adaptar transculturalmente el Test de Adicción a Internet de Young (1998) y evaluar estructuras factoriales anteriormente propuestas. Además, dada su antigüedad, se buscó desarrollar una versión abreviada del instrumento, vigente y con buenas propiedades psicométricas en la cultura chilena. La muestra estuvo constituida por 425 participantes con edades comprendidas entre los 18 y 68 años. A través de un análisis factorial confirmatorio, se constató que ninguna de las soluciones factoriales anteriormente propuestas logró un nivel de buen ajuste a los datos. Mediante un análisis de contenido, se creó una versión abreviada del instrumento con dos factores teóricamente orientados, estructura que presentó tanto un buen ajuste a los datos como una buena consistencia interna.
\end{abstract}

Palabras clave: Test de Adicción a Internet, propiedades psicométricas

\begin{abstract}
Internet addiction is a clinical phenomenon that has raised concerns about the addictive properties of the new information and communication technologies (ICTs). The study aimed to transculturally adapt Young's Internet Addiction Test (1998) and to assess its previously proposed factor structures. Given the test's antiquity, the study also aimed to develop a brief and valid version of it with good psychometric properties. The sample consisted of 425 participants between the ages of 18 and 68 years. Through a series of confirmatory factor analyses, none of the previously proposed factor structures achieved a good level of fitness to the data. A brief version of the instrument with two theoretically oriented dimensions was developed through a content analysis. The proposed structure achieved a good level of internal consistency and fitness to the data.
\end{abstract}

Keywords: Internet Addiction Test, psychometric properties

Agradecimientos: Este estudio recibió apoyo del Fondo de Innovación para la Competitividad (FIC) del Ministerio de Economía, Fomento y Turismo, a través de la Iniciativa Científica Milenio, Proyecto IS130005 y de CONICYT-PCHA beca Doctorado Nacional año 2015.

\footnotetext{
${ }^{1}$ Candidato a Doctor en Psicoterapia, Escuela de Psicología, Pontificia Universidad Católica de Chile. Vicuña Mackenna 4860, Macul, Santiago, Chile e Instituto de Psicología Médica, Centro de Medicina Psicosocial, Hospital Universitario de Heidelberg, Bergheimer Str. 20, Heidelberg, Alemania. Tel.: +562 22425636. Correo: cuhernandez@uc.cl

${ }^{2}$ Profesora Asociada, Escuela de Psicología, Pontificia Universidad Católica de Chile. Vicuña Mackenna 4860, Macul, Santiago, Chile. Tel.: +56223544609. Correo: dvrivera@uc.cl
} 


\section{Introducción}

Desde su creación, la herramienta tecnológica de Internet ha incrementado su alcance e influencia en la sociedad a una tasa impresionante. Esta herramienta ha penetrado al $49.7 \%$ de la población mundial, con una penetración de un 88.1\% en Norteamérica, un $77.4 \%$ en Europa, un $68.1 \%$ en Australia y Oceanía y un $59.6 \%$ en Sudamérica y el Caribe (Internet World Stats, 2017). Dentro de Latinoamérica continental, Chile presenta la segunda mayor tasa de penetración de Internet con un $79.9 \%$ de acceso, superado por Ecuador con un $83.8 \%$ y seguido por Argentina con un 74.9\% (Internet World Stats, 2017).

La conectividad por Internet se ha visto facilitada por la proliferación de aparatos portables (e.g., teléfonos celulares, tabletas, computadores), lo cual facilita su uso en horarios y contextos diversos (e.g., hogar, trabajo, vía pública). Si bien los beneficios de esta herramienta en mejorar la conectividad y la calidad de vida son evidentes, existe cada vez más preocupación sobre comportamientos y consecuencias negativas asociadas al uso de esta tecnología (Blumer, Hertlein, Smith, \& Allen, 2014).

Los comportamientos problemáticos asociado a internet se han denominado de diversas formas, como Uso problemático de Internet, Adicción a Internet, Uso patológico de Internet, Uso excesivo de Internet y Dependencia a Internet (Kelley \& Gruber, 2010; Shapira, et al., 2003; Young, 2010). Aquello que comparten estas definiciones es la incapacidad de la persona de controlar el uso de Internet, lo cual se acompaña de sentimientos de malestar y angustia significativa cuando no se está conectado, junto al deterioro del desempeño en distintas esferas de la vida, como en el estudio, trabajo y/o relaciones sociales (Shapira et al., 2003; Young, 2010).

Independientemente de la denominación del fenómeno, varios estudios han establecido una relación entre el uso excesivo y compulsivo de internet y problemas emocionales, como por ejemplo, sentimientos de soledad (Esen, Aktas,\& Tuncer, 2013; Yao \& Zhong, 2014), ansiedad ante la interacción y las audiencias (Sahin, Korkmaz \& Usta, 2011), impulsividad (Choi, et al., 2014; Dalbudak, et al., 2013), timidez y agresividad
(Odaci \& Çelik, 2013), depresión (Orsal, Orsal, Unsal, \& Ozalp, 2013; Yadav, Banwari, Parmar, \& Maniar, 2013), ansiedad y estrés (Yadav, et al, 2013), percepción negativa sobre el soporte social (Gunuc \& Dogan, 2013), bajo rendimiento académico (Stavropoulos, Alexandraki, \& MottiStefanidi, 2013) y un cronotipo vespertino, asociado a mayor ansiedad y compensación de la deprivación de sueño durante los fines de semana (Lin \& Gau, 2013), asociado en adolescentes a un peor rendimiento académico en matemáticas (Escribano-Barreno \& Díaz-Morales, 2013). También se ha correlacionado positivamente la Adicción a Internet con el tiempo de uso del medio (Khazaal et al., 2008; Young, 2004) y negativamente con la edad, presentándose mayor vulnerabilidad en la población más joven (Khazaal et al., 2008).

El primer instrumento elaborado para evaluar uso problemático de Internet fue el Internet Addiction Test (IAT) de Young (1998), construido en base a los criterios del juego patológico del DSM-IV, bajo la presunción que ambas entidades constituyen adicciones y presentan características conductuales compartidas. El test evalúa el impacto del uso de internet en la rutina diaria, la vida social, la productividad (estudio, trabajo), el sueño y los sentimientos de los usuarios. En Sudamérica, el IAT cuenta con una traducción al español e intento de validación en Colombia (Puerta-Cortés \& Carbonell, 2013; Puerta-Cortés, Carbonell \& Chamarro, 2012). Posteriormente se han desarrollado otros cuestionarios basados en el instrumento original, como son el Cuestionario de Experiencias Relacionadas con Internet -CERI(Beranuy, Chamarro, Graner, \& CarbonellSánchez, 2009) y el Problematic Internet Use Questionnaire - PIUQ - (Demetrovics, Szeredi, \& Rózsa, 2008). Estos cuestionarios comparten varias preguntas con el test original.

El IAT es uno de los instrumentos más utilizados para evaluar Adicción a Internet o Uso Problemático de éste en ambientes clínicos y de investigación (Aboujaoude, 2010). Se han realizado varios intentos de validación en diferentes contextos culturales y características muestrales, alcanzando en general una buena confiabilidad (Jelenchick, Becker, \& Moreno, 2012; Karim \& Nigar, 2013; Khazaal et al., 2008; Korkeila, Kaarlas, Jääskeläinen, Vahlberg, \& 
Taiminen, 2010; Panayides \& Walker, 2012; Puerta-Cortés, et al., 2012; Watters, Keefer, Kloosterman, Summerfeldt, \& Parker, 2013; Widyanto, Griffiths, \& Brunsden, 2011; Widyanto \& McMurran, 2004), pero con inconsistentes estructuras factoriales.

Respecto a lo anterior, el IAT fue evaluado en su estructura por primera vez en el Reino Unido, con una solución de 6 factores (Widyanto \& McMurran, 2004). Posteriores intentos de validación a nivel internacional han encontrado diversas estructuras factoriales, como son la solución de un factor en Francia (Khazaal et al., 2008) y Portugal (Pontes, Patrão, \& Griffiths, 2014); una solución de dos factores en Estados Unidos (Jelenchick et al., 2012), Finlandia (Korkeila et al., 2010) y Canadá (Watters et al., 2013); una solución de tres factores en Colombia (Puerta-Cortés et al., 2013), China (Lai, Mak, Watanabe, Ang, Pang, \& Ho, 2013; Chang \& Man Law, 2008), e Inglaterra (Widyanto et al., 2011); y una solución de cuatro factores en Bangladesh (Karim \& Nigar, 2014).

$\mathrm{La}$ inconsistencia en las estructuras factoriales ha sido atribuida a la diversidad de los participantes utilizados, las diferentes técnicas de reducción elegidas al llevar a cabo los análisis factoriales exploratorios (Watters et al., 2013) y las diferencias culturales que podrían afectar la presentación de los comportamientos problemáticos ante el uso de Internet (Chen \& Nath, 2016). Sin embargo, menor atención se le ha prestado como fuentes de variabilidad y desajuste a las características de los ítems del instrumento y a la posible pérdida de vigencia de alguno de éstos tras su creación el año 1998.

Tomando en consideración la preocupación creciente sobre los problemas asociados al uso de Internet, la acelerada penetración del medio en países de Sudamérica, específicamente en Chile y las diferencias culturales entre ellos, se considera necesario contar con un instrumento válido $\mathrm{y}$ confiable para evaluar la Adicción a Internet y su impacto en la cultura chilena. Dada la inconsistencia de las estructuras factoriales antes propuestas, y de acuerdo a lo anterior, los objetivos del estudio son adaptar el Test de Adicción a Internet (Young, 1998) al español y a la cultura chilena, evaluar el ajuste de las estructuras factoriales establecidas en otros estudios, y proponer una versión abreviada y vigente del instrumento con buenas características psicométricas.

\section{Método}

\section{Participantes}

La muestra estuvo constituida por 425 participantes, un $43.3 \%$ de varones y un $56.7 \%$ de mujeres. La edad se encontró en un rango de 18 a 68 años $(M=26.02, D T=9.96)$. La media de uso semanal de Internet fue de 35.54 horas $(D T=26.02)$. En relación a la ocupación, $2.4 \%$ de los participantes eran estudiantes de educación secundaria, 50.6\% alumnosde educación superior, $28.1 \%$ eran trabajadores dependientes, $10.2 \%$ trabajadores independientes y un $8.7 \%$ estaba desempleado. Casi todos los participantes (96.2\%) tenía conexión a Internet desde su hogar, y un 67.3\% también lo utilizaba para trabajar.

\section{Instrumentos}

Preguntas demográficas y sobre uso de Internet. El cuestionario incluyó preguntas sobre nacionalidad, tiempo viviendo en Chile, sexo, edad, nivel educacional, ocupación principal, tiempo semanal de uso de internet, tener conexión a internet en la casa o el trabajo y porcentaje de uso de Internet en distintas actividades.

Test de Adicción a Internet. El Test de Adicción a Internet (Young, 1998) consiste en 20 ítems medidos en una escala Likert de 6 puntos $(0=n o$ aplica, hasta $5=$ siempre). Evalúa la implicación al Internet de los participantes, además de problemas relacionados al manejo del tiempo, negación del trabajo o los deberes, fantasías sobre el uso de internet y la preferencia de Internet por sobre el contacto social no mediado, entre otras (Ver Anexo 1). El puntaje máximo es de 100 puntos, y mientras más alto el puntaje, mayor es el nivel de problemas asociados. Se utilizó la versión adaptada a Chile para este estudio.

\section{Procedimiento}

Los participantes fueron reclutados a través de Internet, mediante una invitación difundida en foros, blogs y redes sociales tales como Facebook y Twitter, por un período de siete semanas durante el año 2014. Los criterios de inclusión fueron, ser chileno, haber vivido en Chile por al menos dos 
años y ser mayor de edad (18 años para Chile). El cuestionario fue administrado en línea y previo a su acceso, los participantes dispusieron de una carta de consentimiento informado, que contenía una breve explicación del estudio, sus objetivos, y se les informó de la posibilidad de desistir del mismo en cualquier momento sin consecuencia alguna, asegurando el resguardo de su anonimato. Esta información sea compañó de los datos de contacto de los investigadores ante el interés de realizar cualquier consulta. La información fue recopilada a través del Software Survey Monkey, que cuenta con codificación SSL, además de asegurar que ninguna IP fuera descargada en conjunto con los datos.

\section{Traducción y adaptación transcultural del instrumento.}

Si bien existe una traducción sudamericana al español realizada en Colombia por Puerta-Cortés y Carbonell (2013), se decidió realizar el proceso desde el inicio para garantizar una adaptación directa dadas las diferencias de lenguaje cotidiano entre ambos países, y al notarse que la versión colombiana presentó modificaciones mayores en la pregunta tres, y una modificación menor en la pregunta seis.

El instrumento fue traducido al español por dos traductores independientes, siguiendo las recomendaciones de Guillemin, Bombardier y Beaton (1993) para la adaptación transcultural de medidas. Uno de los traductores era parte del equipo de investigación y conocía los objetivos y conceptos subyacentes al material a traducir, de modo de promover así una restitución más confiable del instrumento (Guillemin et al., 1993). El otro traductor no conocía los objetivos y conceptos subyacentes al material, lo cual tenía como objetivo elicitar significados no esperados de la herramienta original. Ambos traductores eran bilingües y tradujeron hacia su lengua materna. Luego fueron realizadas dos retrotraducciones por dos traductores independientes y bilingües, ambos sin conocer los objetivos y conceptos que subyacían al material, para así reducir posibles sesgos. Si bien su lengua nativa era el español, ambos habían vivido en un país de habla inglesa por cerca de 5 años y trabajan actualmente en diferentes agencias de servicios internacionales, presentando un nivel nativo del idioma. Todos los traductores y retro-traductores reportaron sus comentarios, en especial sobre palabras o conceptos difíciles de homologar entre ambos idiomas. Posteriormente se conformó un comité para producir la versión final del instrumento, compuesto por ambos miembros del equipo de investigación y un asesor metodológico, todos bilingües. Siguiendo las recomendaciones de Guillemin et al. (1993), tanto la versión original y las siguientes fueron consideradas como igualmente importantes y abiertas a modificación. Las equivalencias semánticas, idiomáticas, experienciales y conceptuales fueron evaluadas para cada ítem de las cinco versiones (original, traducción 1 y 2 , retrotraducción 1 y 2 ). El instrumento final incluyó las opiniones de los miembros del comité y fue piloteado de acuerdo a las recomendaciones de Gjersing, Caplehorn y Clausen (2010) para la adaptación transcultural de instrumentos de investigación. Treinta voluntarios fueron reclutados a través de redes sociales para parafrasear cada ítem, identificar su comprensión y comentar si consideraron ambigua alguna palabra o concepto. Se agregó al cuestionario una pregunta específica en relación a la palabra Intimidad, debido a que fue detectada como problemática por el comité y los traductores. Dentro de los ajustes semánticos, basados en los resultados del estudio piloto, se agregó en la pregunta 7 , el uso de redes sociales y mensajería instantánea, de modo de adaptar la terminología a los tiempos presentes y mantener su significado, pues han transcurrido 19 años desde la creación del test. Por otra parte, los resultados indicaron que en Chile las personas entienden la palabra intimidad como relaciones sexuales, por lo que se aclaró en el reactivo 3, que se trata de intimidad física y/o emocional (Anexo 1). Salvo estas diferencias, el documento anterior resultó similar a la versión colombiana (Puerta-Cortés \& Carbonell, 2013) e incluyó parte de la modificación que en ésta se realizó a la pregunta seis (traducir "school work" por "deberes académicos") al ser considerada pertinente por el panel de expertos.

\section{Estrategia analítica}

Los análisis estadísticos descriptivos y correlaciones fueron realizados con el lenguaje y ambiente estadístico R (Development Core Team, 
2008). Dada la existencia de las diversas estructuras factoriales descritas anteriormente, se decidió realizar una prueba de aquellas mediante una serie de análisis factoriales confirmatorios (AFC). Para esto se utilizó la librería "Lavaan" para R (Rosseel, 2012) y un estimador de mínimos cuadrados ponderados ajustado por media y varianza (WLSMV) dada la característica ordinal de las respuestas y la robustez de éste para la ausencia de normalidad que habitualmente emerge con preguntas ordinales o categóricas de pocas opciones de respuesta (DiStefano \& Morgan, 2014). El ajuste del modelo a los datos fue evaluado a través de los índices de $\chi^{2}(p>.05)$, CFI yTLI (>.95), RMSEA (<.06) y RMSR (<.08), tomando como indicador de buen ajuste los valores descritos entre paréntesis (Hu \& Bentler, 1999). En el caso del índice RMSEA, se indicó también su intervalo de confianza al $90 \%$. Aun cuando los indicadores CFI y TLI presentan una alta correlación entre sí, se reportaron ambos ya que el segundo tiende a penalizar modelos más complejos (Klyne, 2016). Dado que el instrumento fue construido el año 1998 (Young, 1998), se realizó por parte del equipo de investigación un análisis de contenido de ítems potencialmente problemáticos o que perdieron vigencia tras el rápido cambio de las conductas frente a la tecnología. El objetivo de este proceso fue crear una versión abreviada del Test de Adicción a Internet (TAI) con buenas propiedades psicométricas e ítems vigentes. Los ítems restantes al proceso de eliminación fueron agrupados en dos factores teóricos.

La versión abreviada del TAI fue puesta a prueba a través de un AFC en un proceso de validación cruzada con la mitad de la muestra seleccionada de manera aleatoria. Dicha solución fue revisada para evaluar posibles fuentes de mal ajuste y fue confirmada con la siguiente mitad. Además, se realizó un análisis multi-grupo con la solución seleccionada y la muestra total para estimar su invarianza factorial por sexo, la que fue evaluada a través de una prueba de diferencia de $\chi^{2}$ no significativa y una diferencia de CFI menor a .01 (Cheung \& Rensvold, 2002), ambos indicadores de mantención del ajuste del modelo fijando a igualdad los parámetros seleccionados para ambos grupos. Finalmente se estableció el valor predictivo de la edad y el sexo en las dos variables latentes mediante un modelo de ecuaciones estructurales. Se evaluó la confiabilidad de la escala reducida a través del estadístico Alpha de Cronbach, calculado con la librería "multilevel” para R (Bilese, 2016).

\section{Resultados}

\section{AFC modelos descritos}

Se realizaron diez diferentes AFCs a partir del reporte de las estructuras factoriales descritas en estudios previos, donde ninguna de las estructuras alcanzó un ajuste aceptable en función de los criterios propuestos (Tabla 1). Lo anterior puede indicar problemas tanto en la especificación de los modelos, como en las características de los ítems utilizados (Clark \& Watson, 1995; Hinkin, 1995). Se realizó entonces un análisis de contenido de los ítems con el fin de intentar superar la inconsistencia y mal ajuste de las estructuras probadas, las que no resultaron capaces de reproducir adecuadamente la matriz de correlación observada.

\section{Análisis de contenido y desarrollo de la escala breve}

Se realizó un análisis de contenido de los ítems para eliminar aquellos reactivos potencialmente problemáticos o que hubiesen perdido vigencia. Se buscó eliminar aquellos ítems que tuvieran como objetivo poblaciones específicas, aquellos que resultaran ambiguos, preguntaran por dos fenómenos distintos o que indicaran fuentes de variabilidad no asociadas con un uso adictivo de Internet, ya que éstos podrían incitar una gran heterogeneidad en su interpretación (Clark \& Watson, 1995). Esto podría llevar a una menor validez de contenido al evaluar distintos dominios posibles más allá del de interés (Hinkin, 1995).

Respecto a lo anterior, el ítem 3 fue eliminado debido a que se encuentra condicionado al estar en pareja, mientras que el ítem 4 fue eliminado debido a que hoy en día, tras la emergencia de las redes sociales, no resulta pertinente utilizar la conducta de conocer otras personas online como modo de evaluación de adicción a internet (Khazaal, et al., 2015). Los ítems 6 y 8 fueron eliminados ya que son aplicables a poblaciones distintas (estudiantes, trabajadores y estudiantes/ 
Tabla 1. Índices de Ajuste Análisis Factorial Confirmatorio Escalas Previas

\begin{tabular}{|c|c|c|c|c|c|}
\hline & $\mathrm{x} 2$ & CFI & TLI & RMSEA & RMSR \\
\hline \multicolumn{6}{|l|}{ Un Factor } \\
\hline $\begin{array}{r}\text { Francia y Portugal (Khazaal et al., 2008; Pontes et } \\
\text { al., 2014) }\end{array}$ & $<.05$ & .802 & .777 & .064 & .062 \\
\hline \multicolumn{6}{|l|}{ Dos Factores } \\
\hline USA (Jelenchick, et al., 2012) & $<.05$ & .908 & .896 & .044 & .047 \\
\hline Finlandia (Korleika, et al., 2010) & $<.05$ & .836 & .815 & .058 & .057 \\
\hline Canadá (Watters et al., 2013) & $<.05$ & .940 & .918 & .041 & .037 \\
\hline \multicolumn{6}{|l|}{ Tres Factores } \\
\hline Colombia (Puerta Cortés et al., 2013) & $<.05$ & .892 & .877 & .047 & .050 \\
\hline China (Lai, et al., 2013) & $<.05$ & .887 & .867 & .051 & .050 \\
\hline China (Chang, et al., 2008) & $<.05$ & .881 & .863 & .051 & .051 \\
\hline Inglaterra (Widyanto et al, 2011) & $<.05$ & .826 & .800 & .060 & .057 \\
\hline \multicolumn{6}{|l|}{ Cuatro Factores } \\
\hline Bangladesh (Karim, et al., 2013) & $<.05$ & .844 & .815 & .057 & .054 \\
\hline \multicolumn{6}{|l|}{ Seis Factores. } \\
\hline Reino Unido (Widyanto, et al., 2004) & $<.05$ & .815 & .773 & .064 & .059 \\
\hline
\end{tabular}

Nota. La versión Canadiense (Watters, et al., 2013) utilizó una aproximación bifactorial, mientras que la versión China de Lai, et al., (2013) utilizó una aproximación jerárquica.

trabajadores), a veces mutuamente excluyentes. Las preguntas 9 y 15 fueron eliminadas debido a que preguntan por dos fenómenos distintos dentro de un mismo ítem, aumentando las fuentes de variabilidad y volviendo ambigua su interpretación. Las preguntas 7 y 11 fueron eliminadas, debido a que en el actual contexto altamente penetrado por internet, de alta accesibilidad y requerimiento en ambientes laborales como educacionales, la anticipación de la utilización del medio puede estar ampliamente influenciada por requerimientos externos, y porque los teléfonos inteligentes actuales permiten una conducta de chequeo rápida, no necesariamente problemática. Este punto se refleja en recientes estudios etológicos sobre el establecimiento de conductas de apego a los teléfonos inteligentes similares a las de apego interpersonal (Konok, Gigler, Bereczky, \& Miklósi, 2016). Finalmente, la pregunta 18 fue eliminada pues se consideró que el ocultamiento puede estar asociado a otros fenómenos, como la infidelidad en línea o actividades ilegales, agregando más fuentes de variabilidad y no necesariamente siendo indicativa de una conducta adictiva (Wisman, 2016).

Los 11 ítems restantes fueron teóricamente asignados a dos factores. El primer factor denominado pérdida de control, como su nombre indica, agrupó a los ítems que indican la pérdida de control del uso de internet, ya sea en el tiempo de uso como en las consecuencias de éste. El segundo factor denominado desregulación emocional, agrupó a los ítems que indican un uso de internet asociado a desregulación emocional, incluyendo irritación al ser interrumpido, baja de humor al estar desconectado y evitación de situaciones sociales al preferir estar en internet. Los ítems de la versión abreviada fueron marcados con un asterisco en el Anexo 1.

\section{AFC escala breve}

Para evaluar la estructura factorial del cuestionario propuesto, se realizó un AFC con un método de estimación WLSMV sobre una submuestra aleatoria de 212 casos. El modelo resultante presentó buenos índices de ajuste con un $\chi^{2}(43)=55.951, p>.05$, CFI $=.969$, TLI $=.960$, RMSEA $=.038[90 \%$ CI $=.000-.063]$ y un valor $\mathrm{SRMR}=0.042$. Al realizar un análisis de los residuos, se observó que el ítem 5 presentó comparativamente altos residuos con 6 de los 10 ítems restantes. Además, los índices de modificación indicaban su cambio al factor regulación emocional, cambio que no contó con una justificación teórica, por lo que fue eliminado. El nuevo modelo presentó una mejora en su ajuste con un $\chi^{2}(34)=37.214, \quad p>.05, \quad \mathrm{CFI}=.992$, TLI=.989, RMSEA $=.021[90 \%$ CI $=.000-.056] \mathrm{y}$ un valor $\mathrm{SRMR}=0.035$, además de presentar un 
Tabla 2. Cargas Factoriales y Correlaciones Validación Cruzada

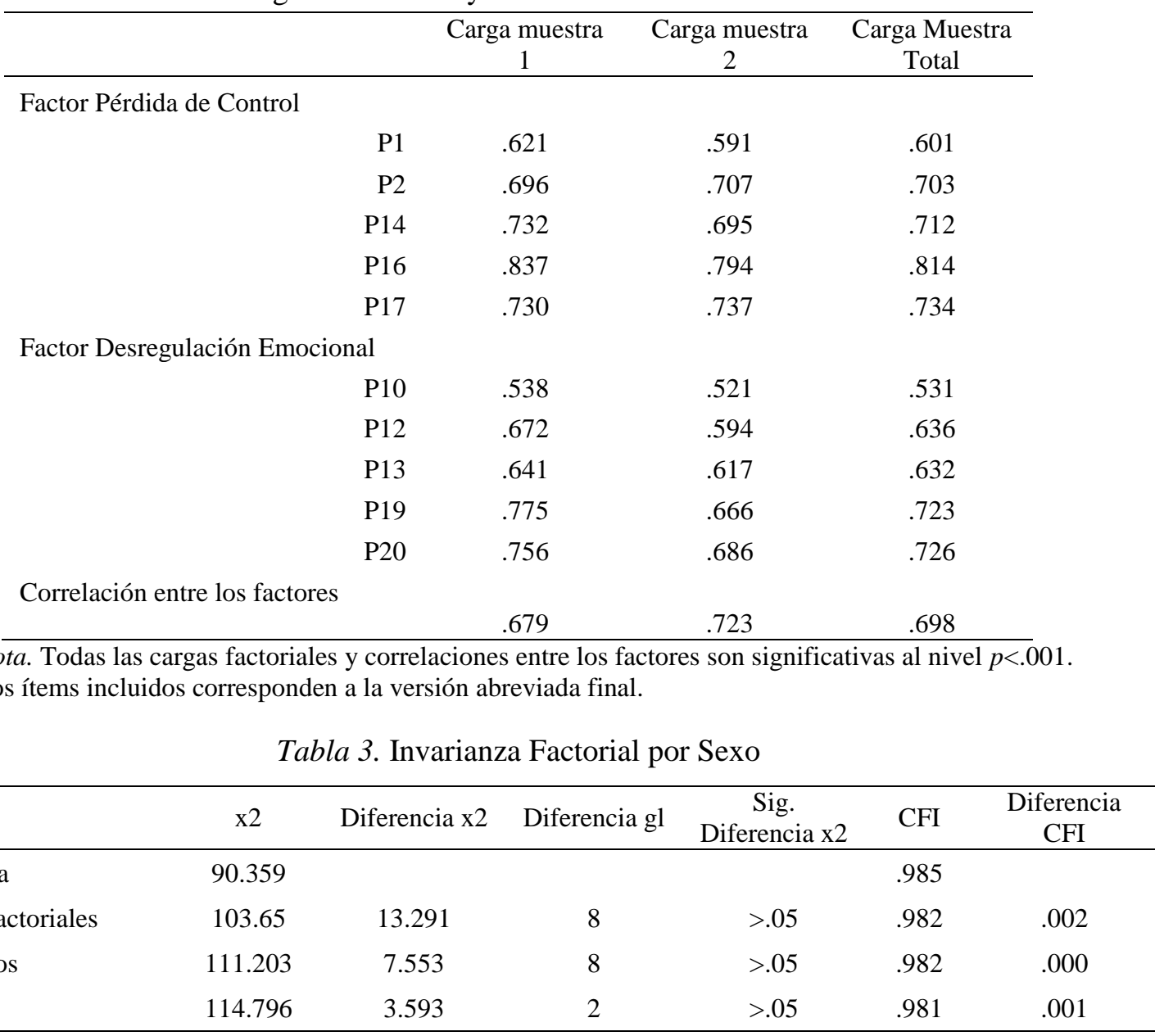

mejor patrón de residuos, por lo que no se justificó la eliminación de más ítems. Se confirmó la estructura factorial modificada con la muestra restante de 213 casos, presentando buenos índices de ajuste con un $\chi^{2}(34)=42.150, p>.05$, CFI $=.978$, TLI=.970, RMSEA $=.034[90 \%$ CI $=.000-.063] \mathrm{y}$ un valor $S R M R=0.042$. Las cargas factoriales de ambas muestras fueron altas y significativas, al igual que la correlación entre los factores (Tabla 2).

Se realizó posteriormente una estimación de un modelo multi-grupo para evaluar la invarianza factorial del modelo propuesto con la muestra total. Para lo anterior se agregaron restricciones de igualdad de parámetros en los siguientes pasos sucesivos: estructura, cargas factoriales, interceptos y residuos. Es posible observar en la tabla 3 que no existen diferencias significativas tanto en los valores $\chi^{2}$, como diferencias mayores a .01 en CFI en todos los pasos, lo que indica la presencia de invarianza estricta: ambos grupos pueden ser comparados en base a este instrumento (Klyne, 2016) y es posible atribuir un mismo significado de los constructos para hombres y mujeres.

En la muestra completa, la escala abreviada total presentó un índice de confiabilidad Alpha de Cronbach de .85, la sub escala de pérdida de control presentó un Alpha de Cronbach de .83, mientras que la escala de desregulación emocional presentó un Alpha de Cronbach de .74.

\section{Relación de escalas con sexo y edad}

Para evaluar la relación de la escala abreviada con el sexo y la edad, se realizó un modelo de ecuaciones estructurales donde el sexo como variable dummy $(0=$ hombre; $1=$ mujer $)$ y la edad de los participantes fueron agregados como predictores de las variables latentes que representan ambos factores. El modelo presentó buenos índices de ajuste con un $\chi^{2}(50)=63.495$, $p>.05, \mathrm{CFI}=.985, \mathrm{TLI}=.980, \mathrm{RMSEA}=.025[90 \%$ $\mathrm{CI}=.000-.043]$ y un valor $\mathrm{SRMR}=0.029$. La edad fue un predictor negativo y significativo tanto del factor de pérdida de control $(\beta=-.427, \mathrm{z}(50)=-$ $8.117, p<.001)$, como del factor de desregulación 
emocional $(\beta=-.278, \mathrm{z}(50)=-5.366, p<.001)$. Por otro lado, el sexo no mostró un efecto significativo tanto para el factor de pérdida de control $(\beta=.037, \mathrm{z}(50)=.753, p>.05)$, como para el factor de desregulación emocional $(\beta=.040$, $\mathrm{z}(50)=.766, p>.05)$. Los valores beta antes reportados se encuentran en su escala estandarizada.

\section{Discusión}

Tomando en consideración la falta de evidencia empírica publicada en relación a la Adicción a Internet en Chile y en Sudamérica, y considerando que dicho país presenta comparativamente una alta tasa de penetración este medio (Internet World Stats, 2017), el presente estudio se enfocó en adaptar transculturalmente el Test de Adicción a Internet (Young, 1998). La evaluación del ajuste de distintas soluciones factoriales propuestas previamente y el análisis de contenido de los ítems, condujo a la eliminación de ítems potencialmente problemáticos, lo cual dio como resultado una escala reducida de dos factores con buenas propiedades psicométricas.

Se probaron diez estructuras factoriales inconsistentes propuestas previamente por la literatura, utilizando un mismo método de estimación, constatándose que ninguna de ellas alcanzó un nivel de buen ajuste. Es probable que dichas inconsistencias y falta de ajuste a los datos se debía a dos grandes problemas. En primer lugar, por la existencia de ítems que pudieron perder vigencia con el exponencial avance y penetración de las TICs en la vida cotidiana de las personas. En segundo lugar, otro grupo de ítems contaba con foco en poblaciones específicas (trabajadores, estudiantes y parejas), además de presentar el potencial de ser interpretados de diversas maneras por los participantes, ya sea por su ambigüedad o por preguntar por dos fenómenos en un mismo reactivo (Clark \& Watson, 1995; Hinkin, 1995). A partir del análisis del contenido de los ítems, y posteriores análisis estadísticos, se propuso una escala reducida con 10 ítems y 2 sub-escalas teóricas, pérdida de control, consistente con la definición operacional de uso problemático o adicción a internet propuesta por Shapira et al. (2003) y desregulación emocional, consistente con el componente de modificación anímica al participar de una determinada actividad, parte del modelo de adicciones de Griffiths (2005). Dicha modificación anímica puede asociarse, por ejemplo, con procesos psicopatológicos asociados al trastorno de ansiedad generalizado, como la evitación de estímulos amenazantes (González, Rovella, Barbenza, \& Rausch, 2012). De hecho, el uso problemático de internet ha sido relacionado positivamente con el hábito de procrastinar (Thatcher, Wretschko, \& Fridkhon, 2007), una forma de comportamiento evitativo. La versión abreviada contó con buenas propiedades psicométricas basadas en su ajuste a los datos, invarianza factorial por sexo y consistencia interna.

Hombres y mujeres no se diferenciaron significativamente en sus puntajes en las subescalas del instrumento, resultado consistente con otros estudios que utilizaron el IAT (Watters, et al., 2013), el PIUQ (Demetrovics, et al., 2008) y el CERI (Beranuy et al., 2009). Este hallazgo puede indicar una creciente equiparación de la utilización de las tecnologías digitales en ambos sexos.

La relación negativa significativa entre edad y puntajes del TAI se encuentra en concordancia con estudios similares (Widyanto et al., 2011, Pontes et al., 2014, Khazaal et al., 2008, PuertaCortés et al., 2013). Frente a lo anterior, es posible considerar una menor edad como un factor de riesgo para el desarrollo de un uso problemático o adictivo de internet. Esto puede ser explicado en parte por su mayor familiaridad con las tecnologías digitales, su constante posibilidad de acceso, los mayores bloques de tiempo desestructurado con los que cuentan y el fomento del uso de internet por parte de las instituciones educacionales (Young, 2004).

Por otro lado, las limitaciones de este estudio se relacionan con su diseño transeccional y su naturaleza en línea, además de presentar una muestra autoseleccionada de participantes, lo que sumado a su tamaño relativamente pequeño, es indicativo de que los resultados deben ser interpretados con precaución. Además, dada la diversidad de posibles experiencias en línea, el Test de Adicción a Internet debe ser utilizado como una medida general, lo que limita su 
potencial de detectar diferencias en aplicaciones específicas. Lo anterior implica que es recomendable que su aplicación sea complementada con medidas que capturen diferencias asociadas a las distintas actividades en línea, como el uso de video juegos, redes sociales y sus distintos sub-tipos, entre otras.

Más allá de sus limitaciones, el presente estudio contribuye con la traducción, adaptación transcultural y evaluación del ajuste de diez intentos de validación previos de uno de los instrumentos más utilizados para evaluar la Adicción a Internet, fenómeno de creciente ubicuidad a nivel internacional, relacionado con diversos problemas psicosociales y asociado a psicopatología ansiosa y depresiva. Además, el presente estudio contribuye con una versión abreviada y teóricamente orientada del instrumento que cuenta con buenas propiedades psicométricas e invarianza factorial entre sexos, sumado a la eliminación de ítems que potencialmente perdieron vigencia con el paso del tiempo. En función de esto, y considerando la presente investigación como un primer paso, futuros estudios se necesitan para explorar el valor predictivo de la versión abreviada, evaluar nuevamente la estructura factorial propuesta, establecer puntajes de corte empíricamente validados, y explorar las distintas facetas de este constructo emergente en la diversidad de contextos de un país y un continente cada vez más permeado con la presencia de Internet $y$ tecnologías de la información. Además, se vuelve necesario evaluar tanto su prevalencia, como su etiopatogenia y el rol que podría cumplir en la génesis o mantención de otros procesos psicopatológicos que afecten significativamente la calidad de vida de quienes los padecen.

\section{Referencias}

Aboujaoude, E. (2010). Problematic Internet use: An overview. World Psychiatry, 9(2), 85-90. doi:10.1002/j.2051-5545.2010.tb00278.x

Beranuy, M., Chamarro, A., Graner, C., \& Carbonell-Sánchez, X. (2009). Validación de dos escalas breves para evaluar la adicción a Internet y el abuso del móvil. Psicothema, 21(3), 480-485. ISSN 0214-9915
Bliese, P. (2016). Multilevel: Multilevel Functions. R package version 2.6. Recuperado de: https://CRAN.R-project.org/package=multilevel

Blumer, M. L., Hertlein, K. M., Smith, J. M., \& Allen, H. (2014). How many bytes does it take? A content analysis of cyber issues in couple and family therapy journals. Journal of Marital and Family Therapy, 40(1), 34-48. doi:10.1111/j.1752-0606.2012.00332.x

Chang, M. K., \& Man Law, S. P. (2008). Factor structure for Young's Internet Addiction Test: A confirmatory study. Computers in Human Behavior, 24(6), 2597-2619. doi:10.1016/j.chb.2008.03.001

Chen, L., \& Nath, R. (2016). Understanding the underlying factors of Internet addiction across cultures: A comparison study. Electronic Commerce Research and Applications, 17, 38-48. doi:10.1016/j.elerap.2016.02.003

Cheung, G. W., \& Rensvold, R. B. (2002). Evaluating goodness-of-fit indexes for testing measurement invariance. Structural Equation Modeling: A Multidisciplinary Journal, 9(2), 233-255. doi:10.1207/s15328007sem0902_5

Choi, J. S., Park, S. M., Roh, M. S., Lee, J. Y., Park, C. B., Hwang, J. Y.,... Jung, H. Y. (2014). Dysfunctional inhibitory control and impulsivity in Internet addiction. Psychiatry Research, 215(2), 424-428. doi:10.1016/j.psychres.2013.12.001

Clark, L. A., \& Watson, D. (1995). Constructing validity: Basic issues in objective scale development. Psychological Assessment, 7(3), 309-319. doi:10.1037/1040-3590.7.3.309

ComScore (2010) Women on the web: How women are shaping the internet. Recuperado de:

http://www.comscore.com/Insights/Presentati ons-and-Whitepapers/2010/Women-on-theWeb-How-Women-are-Shaping-the-Internet.

Dalbudak, E., Evren, C., Topcu, M., Aldemir, S., Coskun, K. S., Bozkurt, M., ... Canbal, M. (2013). Relationship of internet addiction with impulsivity and severity of psychopathology among Turkish university students. Psychiatry Research, 210(3), 1086-1091. doi:10.1016/j.psychres.2013.08.014

Demetrovics, Z., Szeredi, B., \& Rózsa, S. (2008). The three-factor model of Internet addiction: The development of the Problematic Internet 
Use Questionnaire. Behavior Research Methods, $\quad$ 40(2), 563-574. doi: $10.3758 / \mathrm{brm} .40 .2 .563$

DiStefano, C., \& Morgan, G. B. (2014). A comparison of diagonal weighted least squares robust estimation techniques for ordinal data. Structural Equation Modeling: A Multidisciplinary Journal, 21(3), 425-438. doi:10.1080/10705511.2014.915373

Ecribano-Barreno, C., \& Díaz-Morales, J.F. (2013). Rendimiento académico en adolescentes matutinos y vespertinos. Revista Iberoamericana de Diagnóstico y Evaluación - e Avaliação Psicológica, 36, 147-163. doi:10.21865/ridep

Esen, B. K., Aktas, E., \& Tuncer, I. (2013). An Analysis of university students' internet use in relation to loneliness and social self-efficacy. Procedia - Social and Behavioral Sciences, 84, 1504-1508. doi:10.1016/j.sbspro.2013.06.780

Gjersing, L., Caplehorn, J. R., \& Clausen, T. (2010). Crosscultural adaptation of research instruments: Language, setting,time and statistical considerations. BMC Medical Research Methodology, 10(1). doi:10.1186/1471-2288-10-13.

González, M., Rovella, A., Barbenza, C., \& Rausch, L. (2012). Tendencia a la Preocupación y Trastorno de Ansiedad Generalizada en adolescentes: Contribución diferencial de los procesos cognitivos. Revista Iberoamericana de Diagnóstico y Evaluación - e Avaliação Psicológica,33, 31-50.

Guillemin, F., Bombardier, C., \& Beaton, D. (1993). Cross-cultural adaptation of healthrelated quality of life measures: Literature review and proposed guidelines. Journal of Clinical Epidemiology, 46(12), 1417-1432. doi:10.1016/0895-4356(93)90142-n

Gunuc, S., \& Dogan, A. (2013). The relationships between Turkish adolescents' Internet addiction, their perceived social support and family activities. Computers in Human Behavior, 29(6), 2197-2207. doi:10.1016/j.chb.2013.04.011

Griffiths, M. (2005). A "components" model of addiction within a biopsychosocial framework. Journal of Substance Use, 10(4), 191-197. doi:10.1080/14659890500114359
Hinkin, T. (1995). A review of scale development practices in the study of organizations. Journal of Management, 21(5), 967-988. doi:10.1016/0149-2063(95)90050-0

Hu, L., \& Bentler, P. M. (1999). Cutoff criteria for fit indexes in covariance structure analysis: Conventional criteria versus new alternatives. Structural Equation Modeling: A Multidisciplinary Journal, 6(1), 1-55. doi:10.1080/10705519909540118

Internet World Stats (2017) Internet usage and population statistics for South America. Recuperado de http://www.internetworldstats.com/stats $15 . h t m$

Jelenchick, L. A., Becker, T., \& Moreno, M. A. (2012). Assessing the psychometric properties of the Internet Addiction Test (IAT) in US college students. Psychiatry Research, 196(23), 296-301. doi:10.1016/j.psychres.2011.09.007

Kelley, K. J., \& Gruber, E. M. (2010). Psychometric properties of the Problematic Internet Use Questionnaire. Computers in Human Behavior, 26(6), 1838-1845. doi:10.1016/j.chb.2010.07.018

Khazaal, Y., Achab, S., Billieux, J., Thorens, G., Zullino, D., Dufour, M., \& Rothen, S. (2015). Factor structure of the Internet Addiction Test in Online Gamers and Poker Players. JMIR Mental Health, 2(2), e12. doi:10.2196/mental.3805

Khazaal, Y., Billieux, J., Thorens, G., Khan, R., Louati, Y., Scarlatti, E., ... Zullino, D. (2008). French validation of the Internet Addiction Test. Cyber Psychology \& Behavior, 11(6), 703-706. doi:10.1089/cpb.2007.0249

Klyne, R. (2016).Principles and practice of Structural Equation Modeling. The Guildford Press, New York.

Konok., V. Gigler., G. Bereczky, B. M., \& Miklósi, Á. (2016) Humans' attachment to their mobile phones and its relationship with interpersonal attachment style. Computers in Human Behavior, 61, 537-547.

Korkeila, J., Kaarlas, S., Jääskeläinen, M., Vahlberg, T., \& Taiminen, T. (2010). Attached to the web - harmful use of the Internet and its correlates. European Psychiatry, 25(4), 236-241. doi:10.1016/j.eurpsy.2009.02.008 
Lai, C., Mak, K., Watanabe, H., Ang, R., Pang, J., \& Ho, R. (2013) Psychometric properties of the Internet Addiction Test in Chinese adolescents. Journal of Pediatric Psychology, 38(7), 794-807. doi:10.1093/jpepsy/jst022

Lin, Y., \& Gau, S. S. (2013) Association between morningness-eveningness and the severity of compulsive internet use: The moderating role of gender and parenting style. Sleep Medicine, 14(12), 1398-1404. doi:10.1016/j.sleep.2013.06.015

Matsunaga, M. (2010). How to factor-analize your data right: Do's, don'ts, and how-to's. International Journal of Psychological Research, 3(1), 97-110. doi:10.21500/20112084.854

Odac1, H., \& Çelik, Ç. B. (2013). Who are problematic internet users? An investigation of the correlations between problematic internet use and shyness, loneliness, narcissism, aggression and self-perception. Computers in Human Behavior, 29(6), 23822387. doi:10.1016/j.chb.2013.05.026

Orsal, O., Orsal, O., Unsal, A., \& Ozalp, S. S. (2013). Evaluation of Internet Addiction and Depression among University students. Procedia - Social and Behavioral Sciences, 82, 445-454. doi:10.1016/j.sbspro.2013.06.291

Pontes, H. M., Patrão, I. M., \& Griffiths, M. D. (2014). Portuguese validation of the Internet Addiction Test: An empirical study. Journal of Behavioral Addictions, 3(2), 107-114. doi:10.1556/jba.3.2014.2.4

Puerta-Cortés, D. X., \& Carbonell, X. (2013).Uso problemático de Internet en una muestra de estudiantes universitarios colombianos. Avances en Psicología Latinoamericana, 31(3), 620-631.

Puerta-Cortés, D. X., Carbonell, X., \& Chamarro, A. (2012). Análisis de las propiedades psicométricas de la versión en español del Internet Addiction Test. Trastornos Adictivos, 14(4), 99-104. doi:10.1016/s1575-0973(12)70052-1

Karim, A. K. M. R., \& Nigar, N. (2014). The Internet Addiction Test: Assessing its psychometric properties in Bangladeshi culture. Asian Journal of Psychiatry, 10, 7583. doi:10.1016/j.ajp.2013.10.011

R Development Core Team (2008). R: A language and environment for statistical computing. $\mathrm{R}$ Foundation for Statistical Computing, Vienna,
Austria. ISBN 3-900051-07-0, URL http://www.R-project.org.

Rosseel, Y. (2012). Lavaan: AnRPackage for Structural Equation Modeling. Journal of Statistical Software, 48(2). doi:10.18637/jss.v048.i02

Sahin, C., Korkmaz, Ö., \& Usta, E. (2011) The correlation between interaction-audience anxieties and internet addiction levels of adults. Procedia - Social and Behavioral Sciences, $\quad 28, \quad 151-155$. doi:10.1016/j.sbspro.2011.11.030_

Shapira, N. A., Lessig, M. C., Goldsmith, T. D., Szabo, S. T., Lazoritz, M., Gold, M. S., \& Stein, D. J. (2003). Problematic internet use: Proposed classification and diagnostic criteria. Depression and Anxiety, 17(4), 207-216. doi:10.1002/da.10094

Stavropoulos, V., Alexandraki, K., \& MottiStefanidi, F. (2013). Recognizing internet addiction: Prevalence and relationship to academic achievement in adolescents enrolled in urban and rural Greek high schools. Journal of Adolescence, 36(3), 565-576. doi:10.1016/j.adolescence.2013.03.008

Thatcher, A., Wretschko, G., \& Fridjhon, P. (2007). Online flow experiences, problematic Internet use and Internet procrastination. Computers in Human Behavior, 24(5), 22362254. doi:10.1016/j.chb.2007.10.008

Watters, C. A., Keefer, K. V., Kloosterman, P. H., Summerfeldt, L. J., \& Parker, J. D. A. (2013). Examining the structure of the Internet Addiction Test in adolescents: A bifactor approach. Computers in Human Behavior, 29(6), 2294-2302. doi:10.1016/j.chb.2013.05.020

Wisman, M. A. (2016). Discovery of a partner affair and major depressive episode in a probability sample of married or cohabiting adults. Family Process, 55(4), 713-723. doi:10.1111/famp.12185

Widyanto, L., \& McMurran, M. (2004). The Psychometric Properties of the Internet Addiction Test. Cyber Psychology \& Behavior, 7(4), 443-450. doi:10.1089/cpb.2004.7.443

Widyanto, L., Griffiths, M. D., \& Brunsden, V. (2011). A Psychometric comparison of the Internet Addiction Test, the Internet-Related Problem Scale, and Self-Diagnosis. 
Cyberpsychology, Behavior, and Social Networking, 14(3), 141-149. doi:10.1089/cyber.2010.0151

Yadav, P., Banwari, G., Parmar, C., \& Maniar, R. (2013). Internet addiction and its correlates among high school students: A preliminary study from Ahmedabad, India. Asian Journal of Psychiatry, 6(6), 500-505. doi:10.1016/j.ajp.2013.06.004

Yao, M. Z., \& Zhong, Z. (2014). Loneliness, social contacts and Internet addiction: $A$ cross-lagged panel study. Computers in Human Behavior, 30, 164-170. doi:10.1016/j.chb.2013.08.007

Young, K. S. (1998). Internet addiction: The emergence of a new clinical disorder. Cyber Psychology \& Behavior, 1(3), 237-244. doi:10.1089/cpb.1998.1.237

Young, K. S. (2004) Internet addiction: A new clinical phenomenon and its consequences. American Behavioral Scientist, 48, 402-415. doi:10.1177/0002764204270278_

Young, K. S. (2010). clinical assessment of internet-addicted clients. En K. Young \& C. Nabuco de Abreu (Eds.), Internet addiction: A handbook and guide to evaluation and treatment (pp. 19-34). Hoboken, New Jersey: John Wiley \& Sons Inc. doi:10.1002/9781118013991.ch2 


\section{Anexo 1: Versión Chilena Test de Adicción a Internet}

1. ¿Con qué frecuencia se da cuenta que se queda conectado en Internet más tiempo de lo que tenía previsto? *

2. ¿Con qué frecuencia descuida los quehaceres de la casa para pasar más tiempo en línea? *

3. ¿Con qué frecuencia prefiere la emoción de estar conectado a internet antes que la intimidad física y/o emocional con su pareja?

4. ¿Con qué frecuencia forma nuevas relaciones con otros usuarios de internet?

5. ¿Con qué frecuencia quienes lo rodean se quejan por el tiempo que pasa en línea?

6. ¿Con qué frecuencia ve afectadas sus notas o deberes académicos debido al tiempo que pasa en línea?

7. ¿Con qué frecuencia revisa su correo electrónico, Mensajería Instantánea (como Whatsapp) o redes socialesantes de realizar otra cosa que necesite hacer?

8. ¿Con qué frecuencia el tiempo que pasa en internet afecta negativamente su desempeño o productividad en el trabajo?

9. ¿Con qué frecuencia se pone a la defensiva o tiende a ocultar información cuando alguien le pregunta qué hace cuando está en línea?

10. ¿Con qué frecuencia bloquea pensamientos desagradables sobre su vida con pensamientos tranquilizadores o agradables relacionados con internet? $* *$

11. ¿Con qué frecuencia se encuentra a sí mismo anticipando cuándo se conectará nuevamente a internet?

12. ¿Con qué frecuencia teme que la vida sin internet sería aburrida, vacía y triste? **

13. ¿Con qué frecuencia se enoja, grita, o se irrita si alguien lo interrumpe mientras está en línea? **

14. ¿Con qué frecuencia pierde horas de sueño por estar conectado hasta tarde en la noche? *

15. ¿Con qué frecuencia se siente preocupado al estar desconectado de Internet, o fantasea con estar conectado?

16. ¿Con qué frecuencia se encuentra a sí mismo diciendo "solo un par de minutos más" cuando está conectado? *

17. ¿Con qué frecuencia trata de disminuir el tiempo que pasa en Internet y no lo logra?*

18. ¿Con qué frecuencia intenta ocultar cuánto tiempo ha estado en línea?

19. ¿Con qué frecuencia elige pasar más tiempo en línea en lugar de salir con otras personas?**

20. ¿Con qué frecuencia se siente deprimido, de mal humor o nervioso cuando no está conectado a Internet, y se siente mejor cuando se conecta nuevamente? $* *$

Los ítems marcados con asterisco u asteriscos corresponden a la versión abreviada.

* Factor Pérdida de Control.

** Factor Desregulación Emocional. 